\title{
LIQUID HAMMER FOR THE DE-ENERGIZED RADIAL INFLOW TURBINE GENERATOR
}

\author{
Donald R. Smith and Hans E. Kimmel
}

\begin{abstract}
Pressure pulses due to liquid hammer effects are studied for a model of the hydraulic behavior of radial inflow turbines following a power failure during operation. The relevant system of nonlinear differential equations has distinct properties and is of interest in its own right. It is shown that periodic pulsations do not occur for the present time-dependent, spatial-independent model which omits periodic disturbing terms. A pressure pulse occurs and decays quickly as the state of the system surges toward an equilibrium curve which is an attractor in the phase plane. In addition to the nonexistence of periodic pulsations, the path of motion and the location of the limiting state in the phase plane are determined along with the magnitude of the pressure change for the liquid hammer, and a quantitative estimate is given for the pulse decay. The study employs a combination of perturbation techniques and phase plane analysis.
\end{abstract}

\section{Introduction}

The hydraulic behavior of turbines with variable speed is governed by the conservation or balance laws of mass, energy, and momentum. We employ the common assumption that the mass flows across the inlet and the outlet of the turbine are the same, so conservation of mass is automatically satisfied. We use the term "hydraulic" in a generalized sense to refer to any suitable incompressible (or almost incompressible) liquid such as water but also to include other liquids such as oil and liquid natural gas. The present work was initiated in relation to cryogenic pumps for liquid natural gas.

Typical turbines contain many irregular shaped internal channels with variable curved surfaces and variable cross sections. For this reason, the mathematical study of the flow of incompressible fluids through turbines is difficult and is still in its infancy as compared to such well-studied problems as the flow of compressible fluids through nozzles or across airfoils. A simplified black box model for the flow of incompressible liquids through turbines is considered here involving only the average rotation speed of the turbine rotor and the average liquid flow rate through the turbine. In this case, conservation of angular momentum and conservation of energy lead to the nonlinear system (see Kimmel [1]-[3])

$$
\begin{aligned}
& \epsilon \frac{d x}{d t}=y(y-\lambda x)-T(x), \\
& \epsilon \frac{d y}{d t}=-\frac{\alpha x}{\beta y+\gamma}[y(y-\lambda x)-T(x)],
\end{aligned}
$$

Received July 10, 1997, revised January 25, 1998.

1991 Mathematics Subject Classification: 76A99.

Key words and phrases: pressure pulse, radial turbine. 
for real-valued functions $x$ and $y$ (of time $t$ ) which represent, respectively, the rotation speed of the turbine rotor and the liquid flow rate. The constant $\epsilon>0$ is the (scaled) inertia of the turbine rotor, and $\alpha$ and $\beta$ are effective kinetic energy coefficients, respectively, for the rotating turbine and for the liquid flow (cf. (1.10) below). The expression $y(y-\lambda x)$ represents the (scaled) torque due to the turbine shaft where the constant $\lambda$ is a coefficient of shaft torque. The constant $\gamma$ is the average of the upstream and downstream values of the Joukowski coefficient for the differential pressure or hydraulic head $H$. In this regard, we note that conservation of linear momentum follows automatically from the stated two conservation laws of angular momentum and energy along with Joukowski's constitutive equation $\dot{H}=-2 \gamma \dot{y}$ (cf. Stepanoff [6, p.435]). The constants $\epsilon, \beta, \alpha, \lambda$, and $\gamma$ all have positive values, and $\epsilon$ is small for typical radial inflow turbines for common liquids.

The quantity $T(x)$ represents the (scaled) applied torque due to the generator, and it is assumed to be modeled by a suitable specified function of the turbine speed $x$. For example, in the case of a linear generator model, one uses $T=T_{c}+\mu\left(x-x_{s}\right)$ where the constant $x_{s}$ is the synchronous speed of the generator, $T_{c}$ is the constant value of the shaft torque at the synchronous speed, and the constant $\mu$ is a measure of the rate of change of generator torque with respect to speed (at the synchronous speed).

The generator torque function $T(x)=T(x, t)$ also may depend explicitly on certain switching times which are values of $t$ for which the form of the torque function may change abruptly. We consider the case of a single switching time $t_{0}$ and take the applied generator torque as

$$
T(x)= \begin{cases}T_{\operatorname{gen}}(x) & \text { for } t \leq t_{0} \\ 0 & \text { for } t>t_{0}\end{cases}
$$

for a given nonzero function $T_{\text {gen }}$. The time $t_{0}$ corresponds to a power failure at which time the generator is de-energized and the previously nonzero generator torque $T_{\text {gen }}$ is suddenly switched to zero. The time $t_{0}$ is called the disconnect time since the generator may be considered to be disconnected from the turbine at this time.

During steady-state energized operation prior to the disconnect time, the driven turbine is assumed to operate at a constant equilibrium state

$$
x(t)=x_{0} \quad \text { and } \quad y(t)=y_{0} \quad \text { for } \quad t \leq t_{0}
$$

for suitable fixed constants $x_{0}$ and $y_{0}$ which are the coordinates of the equilibrium state in the $(x, y)$ phase plane. The equilibrium state $\left(x_{0}, y_{0}\right)$ corresponds to a balance of (the generally opposing) shaft and generator torques, so the point $\left(x_{0}, y_{0}\right)$ in the phase plane satisfies the equilibrium equation (see (1.1), (1.2))

$$
y_{0}\left(y_{0}-\lambda x_{0}\right)=T_{\text {gen }}\left(x_{0}\right)
$$

where the generator torque is nonzero prior to the disconnect time (during the powered or energized operation),

$$
T_{\text {gen }}\left(x_{0}\right) \neq 0 .
$$

The constant equilibrium operating state $\left(x_{0}, y_{0}\right)$ of $(1.3)-(1.5)$ is said to be a rated state for the energized turbine. For the radial inflow turbine, we make the customary 
assumption that the rated state is in the first quadrant in the phase plane,

$$
x_{0} \geq 0 \text { and } y_{0}>0
$$

where there is no loss in allowing $x_{0}$ to vanish as long as the liquid flow rate is positive, $y_{0}>0$. In practice, for the radial inflow ${ }^{1}$ turbine, one sometimes also stipulates the condition $y_{0}>\lambda x_{0}$ in addition to (1.6), but here we consider the three broader cases indicated by (3.2)-(3.4) below.

We consider a power failure corresponding to a disconnection of the generator from the turbine at the time $t_{0}$ as in (1.2). Following the disconnect time, the system begins to move away from the previous rated equilibrium state $\left(x_{0}, y_{0}\right)$ under the influence of a modified system (1.1) which now becomes

subject to the initial conditions

$$
\begin{aligned}
& \epsilon \frac{d x}{d t}=y(y-\lambda x), \\
& \epsilon \frac{d y}{d t}=-\frac{\alpha x y(y-\lambda x)}{\beta y+\gamma} \quad \text { for } \quad t>t_{0},
\end{aligned}
$$

$$
x=x_{0} \text { and } y=y_{0} \text { at } t=t_{0}
$$

where the initial state $\left(x_{0}, y_{0}\right)$ satisfies the conditions of (1.4)-(1.6). (The quantities $x(t)$ and $y(t)$ are assumed to be continuous across the disconnect time.)

The system (1.7) (or (1.1)) can be solved explicitly by quadrature. For example, the flow rate $y=y(t)$ can be studied for (1.7)-(1.8) with the relation

$$
t=t_{0}+\mathcal{T}(y(t)) \quad \text { for } \quad t \geq t_{0}
$$

where the function $\mathcal{T}=\mathcal{T}(y)$ is given as

$$
\mathcal{T}(y):=-\epsilon \int_{y_{0}}^{y} \frac{(\beta s+\gamma) d s}{\alpha \mathcal{N}(s) s(s-\lambda \mathcal{N}(s))}
$$

with $\mathcal{N}(s)$ defined as

$$
\mathcal{N}(s):=\frac{1}{\sqrt{\alpha \beta}} \sqrt{\alpha \beta x_{0}^{2}+\left(\beta y_{0}+\gamma\right)^{2}-(\beta s+\gamma)^{2}}
$$

for values of $s$ near $y_{0}$. The speed $x(t)$ is obtained explicitly from the flow value $y(t)$, and it is delivered by the function $\mathcal{N}$ evaluated at $s=y(t)$ as

$$
x(t)=\mathcal{N}(y(t))
$$

where the positive square root is used in (1.9) in accordance with (1.6). This solution algorithm makes use of the first integral (2.3) below. We do not pursue this approach here; in the following, we give a direct study of the relevant problem based on a combination of phase plane analysis and perturbation techniques.

The differential pressure or hydraulic head from the inlet to the outlet of the turbine is denoted as $H$. It represents the potential energy associated with the contact or stress forces in the fluid. Conservation of energy requires that this hydraulic head potential energy must balance the sum of the kinetic energies of the flow and of the rotating turbine with

$$
H=\alpha x^{2}+\beta y^{2}
$$

\footnotetext{
${ }^{1}$ The case $y_{0}<0$ corresponds to a radial outflow turbine which is known to have low efficiency and is not considered here.
} 
A pressure pulse or pressure jump known as a liquid hammer is generated in the fluid by the power failure at time $t=t_{0}$, and it is important to predict the magnitude and decay properties of this hammer. One also wishes to know whether such pressure pulses due to a hammer may or may not persist in a periodic fashion.

In Section 2, we give a first (energy) integral for the system (1.1) (including (1.7)), and we show that periodic pressure pulsations do not occur for the present model in the case of a power failure. In Section 3, we show that the equilibrium line

$$
y=\lambda x
$$

is an attractor for (1.7) in the first quadrant of the phase plane, which means that every solution moves asymptotically toward a (solution dependent) limiting point $\left(x^{*}, y^{*}\right)$ located on the line (1.11). We obtain explicit formulas for the coordinates of this limiting point. For small $\epsilon$, the state of the system surges rapidly from the initial state $\left(x_{0}, y_{0}\right)$ to close proximity of the limiting state $\left(x^{*}, y^{*}\right)$ within a small time period that is proportional to $\epsilon$. The pressure change due to the pulse is denoted as $\Delta H$ and is given as

$$
\Delta H=\alpha\left[\left(x^{*}\right)^{2}-x_{0}^{2}\right]+\beta\left[\left(y^{*}\right)^{2}-y_{0}^{2}\right] .
$$

An explicit formula is given for $\Delta H$ in terms of the data in Section 3 where it is shown that $\Delta H$ is proportional to the difference $y_{0}-y^{*}$ between the rated value and the limiting value of the liquid flow rate, with the constant of proportionality given by the negative of twice Joukowski's coefficient of differential pressure. The solutions of the systems (1.1) or (1.7) depend on the scaled time variable $t / \epsilon$. Quantitative error estimates are given in Section 4 which show that the pressure pulse decays exponentially fast with a time constant proportional to $\epsilon$.

\section{The energy integral}

Assuming that the rotation speed $x=x(t)$ and the liquid flow rate $y=y(t)$ are continuously differentiable during a period of operation of the turbine, the following first integral or energy relation (2.1) is obtained directly from (1.1).

Theorem 1. The rotation speed $x=x(t)$ and the flow rate $y=y(t)$ for the turbine system (1.1) satisfy

$$
\alpha \beta x^{2}+(\beta y+\gamma)^{2}=\text { constant }
$$

during any time interval for which the turbine undergoes a smooth operation, where the constant on the right-hand side of (2.1) can be evaluated as

$$
\text { constant }=\alpha \beta x_{1}^{2}+\left(\beta y_{1}+\gamma\right)^{2}
$$

with $x_{1}$ and $y_{1}$ taken to be the values of the rotation speed and flow at any fixed instant during the operation of the turbine.

Proof. Upon differentiation of the expression $\alpha \beta x(t)^{2}+(\beta y(t)+\gamma)^{2}$, with (1.1), one finds directly the result

$$
\frac{d}{d t}\left[\alpha \beta x(t)^{2}+(\beta y(t)+\gamma)^{2}\right]=0
$$

for all $t$ during the flow, from which the stated result follows immediately. 


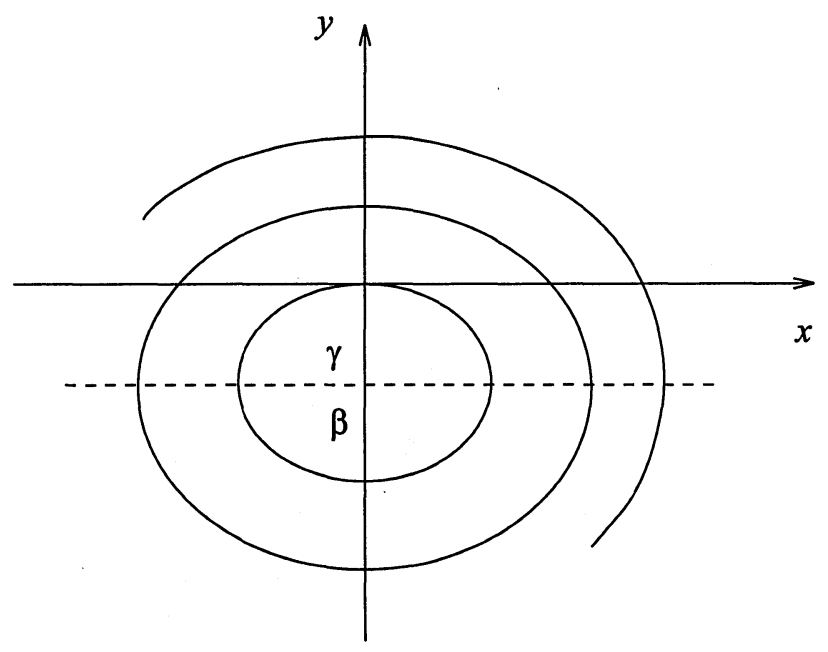

Figure 1

For any fixed positive values of $\alpha$ and $\beta$, the energy equation (2.1) characterizes an ellipse in the $(x, y)$ phase plane for each specified positive value of the constant on the right-hand side of (2.1). There is a unique ellipse (2.1) passing through each point in the phase plane, with constant given by (2.2) as indicated in Figure 1. Theorem 1 states that each solution of (1.1) will move along the appropriate ellipse (2.1) in the phase plane during the operation of the turbine-provided the state of the system is not forced to jump or switch from one ellipse to another by an externally imposed change in (or addition or modification to) any of the model properties.

For the power failure case, we have

Theorem 2. For the initial value problem (1.7) and (1.8), the solution functions $x=x(t)$ and $y=y(t)$ satisfy

$$
\alpha \beta x^{2}+(\beta y+\gamma)^{2}=\alpha \beta x_{0}^{2}+\left(\beta y_{0}+\gamma\right)^{2}
$$

for $t \geq t_{0}$.

Proof. (2.3) follows directly from Theorem 1 and the initial conditions (1.8).

Theorem 2 implies directly the following important result on the nonexistence of periodic solutions.

Theorem 3. The system of differential equations (1.7) has no nontrivial (i.e., nonconstant) periodic solutions.

Proof. A nonconstant periodic solution must follow a path in the phase plane that returns to its initial state after each period. The solution path also must coincide with a portion (or all) of the appropriate ellipse (2.1) or (2.3). Such a periodic solution cannot oscillate back and forth between two distinct points along a segment of the ellipse (without making a complete traversal of the ellipse) because otherwise there would be points on this segment for which the solution would have different (opposite) signs for $\dot{x}$ and for $\dot{y}$ at the same point with the motion reversed, coming and going in different directions. But this would contradict the fact that the system (1.7) 


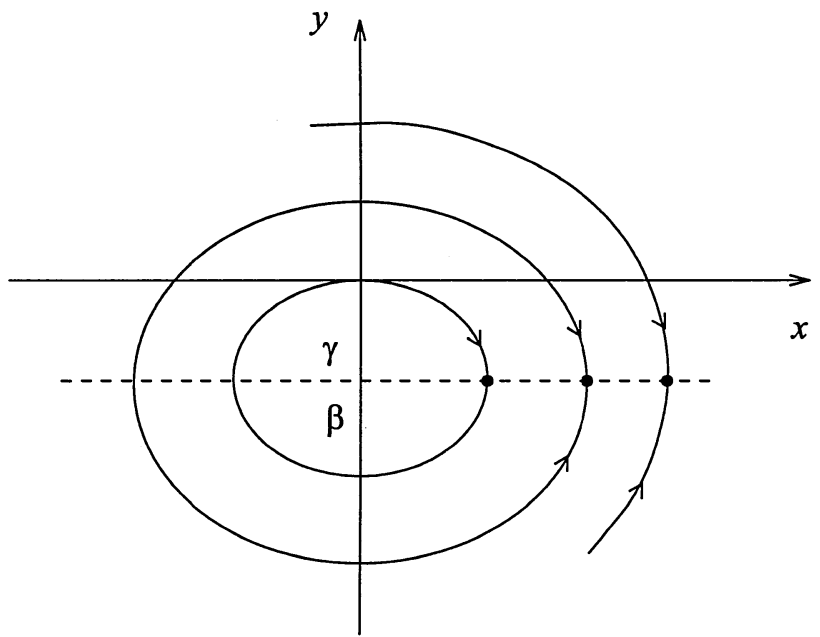

Figure 2

determines unique values for $\dot{x}$ and $\dot{y}$ at each point in the phase plane. Hence any such nontrivial periodic solution must correspond to (successive) complete traversals around the appropriate ellipse (2.1) with no retracements.

We now show that no such "complete traversal" solution can occur. To this end, note that every ellipse (2.1) intersects the line $\beta y+\gamma=0$ at a point $(x, y)$ in the fourth quadrant with $x>0, y<0$. However, in the fourth quadrant, there holds $y-\lambda x<0$, and then

$$
-\alpha x y(y-\lambda x)<0 \quad \text { for all }(x, y) \text { in the fourth quadrant }
$$

where $x>0$ and $y<0$. It follows from (2.4) and the second equation of (1.7) that, in the fourth quadrant, there holds

$$
\begin{cases}\dot{y}<0 & \text { for } y>-\gamma / \beta, \\ \dot{y}>0 & \text { for } y<-\gamma / \beta .\end{cases}
$$

That is, in the fourth quadrant, every solution moves along the appropriate ellipse toward the line $y=-\beta / \gamma$, both from above and from below this line, as indicated in Figure 2. Hence no solution can cross this line $y=-\beta / \gamma$, and no solution can traverse its ellipse completely without retracement.

\section{The attracting equilibrium curve}

The line $y=\lambda x$ is a line of equilibrium points for (1.7), which means that any point $\left(x_{0}, y_{0}\right)$ on this line in the phase plane is a fixed point solution. That is, the constant functions

$$
x(t) \equiv x_{0} \quad \text { and } \quad y(t) \equiv y_{0} \quad \text { for all } t
$$

provide solutions for (1.7) if

$$
y_{0}=\lambda x_{0} .
$$




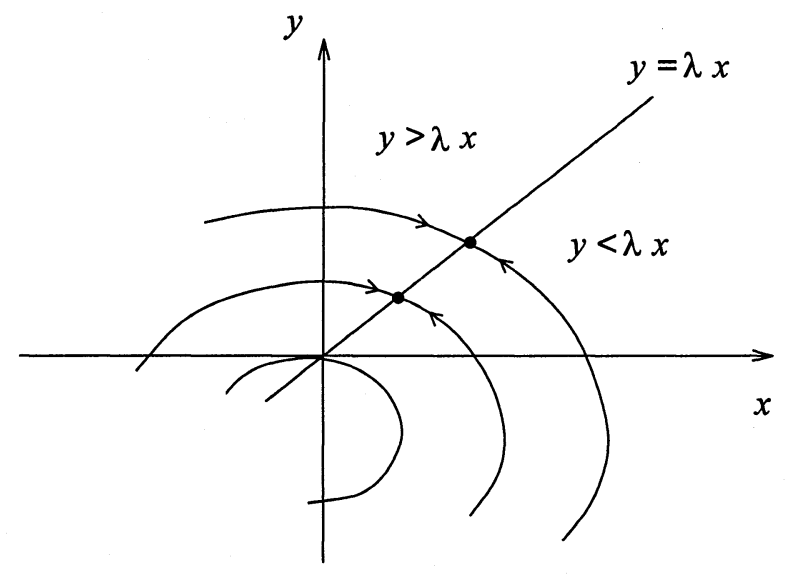

FIGURE 3

In this case not only is the generator torque zero (disconnected power), but also there is no load because the shaft torque $y(y-\lambda x)$ is also zero. ${ }^{2}$

We consider initial states in the first quadrant. In addition to (3.2), there are two other possibilities, namely

$$
y_{0}>\lambda x_{0}
$$

and

$$
y_{0}<\lambda x_{0}
$$

where in both cases we assume ${ }^{3}$

$$
x_{0}>0 \text { and } y_{0}>0 .
$$

The differential equations of (1.7) yield directly

$$
\dot{x}\left(t_{0}\right)>0 \text { and } \dot{y}\left(t_{0}\right)<0 \text { in the case (3.3), }
$$

and

$$
\dot{x}\left(t_{0}\right)<0 \text { and } \dot{y}\left(t_{0}\right)>0 \text { in the case (3.4). }
$$

In the first quadrant of the phase plane, with increasing $t$, the system moves "down" along the ellipse (2.1)-(2.2) toward the line $y=\lambda x$ in the case (3.3), while the system moves "up" along the ellipse toward $y=\lambda x$ in the other case (3.4), as indicated in Figure 3.

\footnotetext{
${ }^{2}$ The shaft torque is zero also for $y=0$, so the line $y=0$ in the phase plane is also a line of equilibrium points for (1.7). These latter equilibrium points are of no particular importance in the case considered here with the initial state taken to be in the first quadrant.

${ }^{3}$ The case $x_{0}=0$ also can be included provided $y_{0}>0$ holds. The $x$-axis, $y=0$, also consists of equilibrium points for (1.7), but such equilibrium points $\left(x_{0}, 0\right)$ are unstable for $x_{0} \neq 0$ and are excluded by (1.6).
} 
A routine calculation shows that the intersection point $\left(x^{*}, y^{*}\right)$ of the line $y-\lambda x=0$ and the ellipse (2.3) in the first quadrant has coordinates

$$
\begin{aligned}
& x^{*}=x^{*}\left(x_{0}, y_{0}\right)=\frac{\sqrt{\lambda^{2}\left[\alpha \beta x_{0}^{2}+\left(\beta y_{0}+\gamma\right)^{2}\right]+\alpha\left[\alpha x_{0}^{2}+\beta y_{0}^{2}+2 \gamma y_{0}\right]}-\lambda \gamma}{\alpha+\beta \lambda^{2}}, \\
& y^{*}=y^{*}\left(x_{0}, y_{0}\right)=\lambda x^{*}\left(x_{0}, y_{0}\right) .
\end{aligned}
$$

In the following we also use the identity

$$
\epsilon \frac{d}{d t}\left[\frac{x(t)}{\beta y(t)+\gamma}\right]=\frac{\alpha \beta x(t)^{2}+(\beta y(t)+\gamma)^{2}}{(\beta y(t)+\gamma)^{3}} y(t)[y(t)-\lambda x(t)],
$$

which can be checked by direct differentiation on the left-hand side of (3.9) using the equations of (1.7).

Theorem 4. The equilibrium line $y=\lambda x$ is an attractor in the first quadrant for the system (1.7). For initial states $\left(x_{0}, y_{0}\right)$ in the first quadrant, the solution state $(x(t), y(t))$ for the initial value problem (1.7)-(1.8) moves along the ellipse (2.3) toward the limiting point (3.8) located at the point of intersection of the ellipse and the attracting line $y=\lambda x$. In the case $y_{0}=\lambda x_{0}$, the solution state is constant and coincides with the limiting state $x=x_{0}=x^{*}$ and $y=y_{0}=y^{*}$. Otherwise (if $y_{0} \neq \lambda x_{0}$ ) the solution state approaches the limiting point $\left(x^{*}, y^{*}\right)$ asymptotically along the ellipse (2.3), but the state of the system never arrives at this limiting point in any finite time. Moreover,

$$
[y(t)-\lambda x(t)] \cdot\left[y_{0}-\lambda x_{0}\right]>0 \text { for all } t \geq t_{0} \quad \text { if } \quad y_{0} \neq \lambda x_{0},
$$

so the sign of $y(t)-\lambda x(t)$ is invariant and coincides always with the initial sign of $y_{0}-\lambda x_{0}$. Finally, if $y_{0} \geq \lambda x_{0}$, then

$$
\begin{gathered}
\frac{\alpha x_{0}}{\beta y_{0}+\gamma} \leq \frac{\alpha x(t)}{\beta y(t)+\gamma} \leq \frac{\alpha x^{*}}{\beta y^{*}+\gamma} \quad \text { and } \\
x_{0} \leq x(t) \leq x^{*}, \quad y^{*} \leq y(t) \leq y_{0} \quad \text { for all } t \geq t_{0}
\end{gathered}
$$

while if $y_{0} \leq \lambda x_{0}$, then

$$
\begin{gathered}
\frac{\alpha x^{*}}{\beta y^{*}+\gamma} \leq \frac{\alpha x(t)}{\beta y(t)+\gamma} \leq \frac{\alpha x_{0}}{\beta y_{0}+\gamma} \quad \text { and } \\
x^{*} \leq x(t) \leq x_{0}, \quad y_{0} \leq y(t) \leq y^{*} \quad \text { for all } t \geq t_{0}
\end{gathered}
$$

Proof. The standard uniqueness theorem for the initial value problem is used several times in the following discussion. First, the case $y_{0}=\lambda x_{0}$ is trivial since the constant state (3.1) is a solution (hence the only solution) of (1.7)-(1.8) in this case.

If the initial state in the first quadrant satisfies $y_{0}-\lambda x_{0}>0$, then (2.3) and (3.6) imply that the solution moves along the ellipse initially towards the limiting point (3.8) on the line $y-\lambda x=0$. The limiting point (3.8) is itself a constant equilibrium solution of the system, so the uniqueness theorem guarantees that the solution point beginning at $\left(x_{0}, y_{0}\right) \neq\left(x^{*}, y^{*}\right)$ never arrives at the limiting point in finite time in this case. Moreover, in this case, the differential equations of (1.7) guarantee that the solution satisfies $\dot{x}>0$ and $\dot{y}<0$ for all $t \geq t_{0}$, so the solution continues to move toward the limiting point which is the nearest equilibrium point (in the direction of motion for the system) on the ellipse. It follows with the uniqueness theorem that $y(t)-\lambda x(t)>0$ for $t \geq t_{0}$ holds, which proves the validity of (3.10) in this case. The results of (3.11) for $\alpha x /(\beta y+\gamma)$ follow from (3.9) since (3.9) and (3.10) imply that $x(t) /(\beta y(t)+\gamma)$ is 
a strictly increasing function of $t$ in the present case with $y_{0}-\lambda x_{0}>0$. The results of (3.11) for $x(t)$ and $y(t)$ follow directly from (1.7) and (3.10). This completes the proof of the theorem in the case (3.3), and similar arguments handle the other case (3.4).

According to (1.12), the change $\Delta H$ in the hydraulic head due to the liquid hammer appears to depend quadratically on the coordinates of the rated and limiting points in the phase plane. However, upon inserting (3.8) into (1.12), a routine calculation shows that this pressure change actually depends linearly on the difference between the liquid flow rates at the rated state and at the limiting state, with

$$
\Delta H=-2 \gamma \cdot\left[y^{*}-y_{0}\right],
$$

which is in agreement with Joukowski's relation $(d / d t) H=-2 \gamma y$. In particular, $\Delta H=0$ holds if and only if either $\gamma=0$ or $y_{0}=\lambda x_{0}$ (with $y^{*}=y_{0}$ ).

The (scaled) inertia $\epsilon$ of the turbine rotor is small for typical turbines as considered here. In these cases, the system (1.7) is singularly perturbed, and it is shown in Section 4 that the solution point $(x(t), y(t))$ moves quickly to a close proximity of the appropriate limiting point $\left(x^{*}, y^{*}\right)$ on the equilibrium line. The limiting point is itself a constant state fixed point solution of the system (1.7). As noted earlier, it follows from the standard uniqueness theorem for ODE initial value problems that the solution points $(x(t), y(t))$ corresponding to the cases (3.3) and (3.4) never actually arrive at the limiting point $\left(x^{*}, y^{*}\right)$ in any finite time in an exact mathematical sense. However, the system moves quickly to very close proximity of the limiting point when $\epsilon$ is small, so that, in a practical sense, the state of the system can be considered to arrive essentially at the equilibrium limiting point in a short time that is proportional to the inertia $\epsilon$.

\section{Pulse decay}

When we wish to emphasize the dependence of the solution on the initial state $\left(x_{0}, y_{0}\right)$ for the initial value problem (1.7)-(1.8), we denote the solution functions as

$$
x(t)=x\left(t ; x_{0}, y_{0}\right) \quad \text { and } \quad y(t)=y\left(t ; x_{0}, y_{0}\right) .
$$

The following theorem, which gives estimates on the differences $x\left(t ; x_{0}, y_{0}\right)-x^{*}$ and $y\left(t ; x_{0}, y_{0}\right)-y^{*}$ between the solution functions and the coordinates of the corresponding limiting equilibrium point $\left(x^{*}, y^{*}\right)$, is proved using techniques from perturbation theory (cf. Smith [5]).

The linearization of (1.7) about the limiting equilibrium point $\left(x^{*}, y^{*}\right)$ has one negative eigenvalue and one zero eigenvalue, so $\left(x^{*}, y^{*}\right)$ is not a hyperbolic equilibrium point for (1.7). A center manifold for $\left(x^{*}, y^{*}\right)$ is the attractor line $y=\lambda x>0$ (consisting itself solely of equilibrium points), while the stable manifold for $\left(x^{*}, y^{*}\right)$ is the portion of the ellipse (2.3) lying in the upper half-plane and passing through $\left(x^{*}, y^{*}\right)$; cf. Perko [4].

Theorem 5. Assume that the initial state $\left(x_{0}, y_{0}\right)$ lies in the first quadrant (so that (3.5) holds) with $y_{0} \neq \lambda x_{0}$, and assume that the parameters $\epsilon, \alpha, \beta, \gamma$, and $\lambda$ appearing in the system (1.7) are all positive. Then the solution functions (4.1) for the initial 


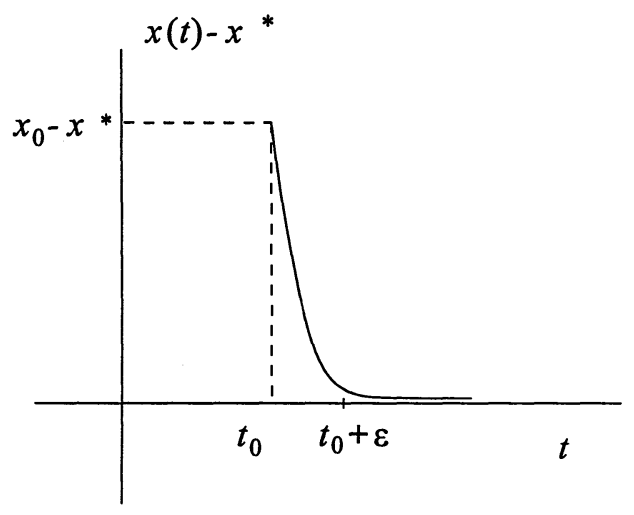

FIgURE 4

value problem (1.7)-(1.8) satisfy the estimates

$$
\begin{aligned}
& \left|x\left(t ; x_{0}, y_{0}\right)-x^{*}\right| \leq \xi \cdot\left|y_{0}-\lambda x_{0}\right| \cdot e^{-\kappa\left(t-t_{0}\right) / \epsilon}, \\
& \left|y\left(t ; x_{0}, y_{0}\right)-y^{*}\right| \leq \eta \cdot\left|y_{0}-\lambda x_{0}\right| \cdot e^{-\kappa\left(t-t_{0}\right) / \epsilon} \quad \text { for } t \geq t_{0},
\end{aligned}
$$

for positive constants $\xi$ and $\eta$ and for a positive decay constant $\kappa$ that may be given as

$$
0<\kappa:= \begin{cases}\left(\frac{\alpha x_{0}}{\beta y_{0}+\gamma}+\lambda\right) y^{*} & \text { if } y_{0}>\lambda x_{0}, \\ \left(\frac{\alpha x^{*}}{\beta y^{*}+\gamma}+\lambda\right) y_{0} & \text { if } y_{0}<\lambda x_{0},\end{cases}
$$

where the limiting values $x^{*}=x^{*}\left(x_{0}, y_{0}\right)$ and $y^{*}=y^{*}\left(x_{0}, y_{0}\right)$ are given by (3.8), and where the positive constants $\xi$ and $\eta$ may be taken as

$$
\xi=\frac{1}{\lambda} \text { and } \eta=1 .
$$

The quantity $\left|y_{0}-\lambda x_{0}\right|$ appearing as a factor on the right-hand side of the estimates of (4.2) may be replaced with $\left|T_{g e n}\left(x_{0}\right) / y_{0}\right|$ as in (1.4).

Before giving the proof of Theorem 5 we note that the estimates of (4.2) imply that the functions $x(t)$ and $y(t)$ decay rapidly to their constant limiting values $x^{*}$ and $y^{*}$ for small $\epsilon>0$ and $t>t_{0}$. Indeed,

$$
\int_{t_{0}}^{\infty} e^{-\kappa\left(t-t_{0}\right) / \epsilon} d t=\frac{\epsilon}{\kappa}
$$

so (4.2) implies

$$
\int_{t_{0}}^{\infty}\left|x(t)-x^{*}\right| d t \leq \frac{\xi \cdot\left|y_{0}-\lambda x_{0}\right|}{\kappa} \cdot \epsilon
$$

along with an analogous result for $y(t)$. Hence, for $t \geq t_{0}$, the area between the graphs of $x(t)$ and $x^{*}$ (or the area between $y(t)$ and $y^{*}$ ) is small, of order $\epsilon$, as indicated in Figure 4 for $x(t)$. 
Proof of Theorem 5. For brevity, introduce the difference functions $X$ and $Y$ defined as

$$
\begin{gathered}
X(t) \equiv X\left(t ; x_{0}, y_{0}\right):=x\left(t ; x_{0}, y_{0}\right)-x^{*} \\
Y(t) \equiv Y\left(t ; x_{0}, y_{0}\right):=y\left(t ; x_{0}, y_{0}\right)-y^{*}
\end{gathered}
$$

with $x=x^{*}+X$ and $y=y^{*}+Y$ and where $x^{*}=x^{*}\left(x_{0}, y_{0}\right)$ and $y^{*}=y^{*}\left(x_{0}, y_{0}\right)$ are given by (3.8). Insert (4.5) into (1.7) and use the relation $y^{*}=\lambda x^{*}$ to find the differential equations

$$
\begin{aligned}
& \epsilon \frac{d X}{d t}=y(t)(Y-\lambda X) \\
& \epsilon \frac{d Y}{d t}=-\frac{\alpha x(t) y(t)}{\beta y(t)+\gamma}(Y-\lambda X)
\end{aligned}
$$

for $t>t_{0}$

$$
X=x_{0}-x^{*} \quad \text { and } \quad Y=y_{0}-y^{*} \quad \text { at } \quad t=t_{0} .
$$

Multiply the first equation of (4.6) by $\lambda$ and subtract the resulting equation from the second equation of $(4.6)$ to find

$$
\epsilon \frac{d}{d t}(Y-\lambda X)=-\left[\frac{\alpha x(t)}{\beta y(t)+\gamma}+\lambda\right] y(t)(Y-\lambda X) \text { for } t>t_{0}
$$

subject to the initial condition

$$
Y-\lambda X=y_{0}-\lambda x_{0} \text { at } t=t_{0}
$$

where (4.7) has been used along with the relation $y^{*}=\lambda x^{*}$. The equations (4.8) and (4.9) yield directly the equivalent integral relation

$$
\frac{Y(t)-\lambda X(t)}{y_{0}-\lambda x_{0}}=e^{-\frac{1}{\epsilon} \int_{t_{0}}^{t} A(s) d s}>0 \quad \text { for } \quad t \geq t_{0} \quad\left(\text { with } y_{0}-\lambda x_{0} \neq 0\right)
$$

for the function $Y-\lambda X$ where the function $A(t)$ (which depends on the considered solution functions $x(t)$ and $y(t))$ is given as

$$
A(t):=\left(\frac{\alpha x(t)}{\beta y(t)+\gamma}+\lambda\right) y(t) \text { for } t \geq t_{0} .
$$

We first consider the case (3.3) with $y_{0}-\lambda x_{0}>0$. Then Theorem 4 leads to the inequality

$$
\left(\frac{\alpha x(t)}{\beta y(t)+\gamma}+\lambda\right) y(t) \geq\left(\frac{\alpha x_{0}}{\beta y_{0}+\gamma}+\lambda\right) y^{*} \stackrel{(4.3)}{=} \kappa \text { for } t \geq t_{0}
$$

which with (4.11) gives $-\frac{1}{\epsilon} A(t) \leq-\kappa / \epsilon<0$ for $t \geq t_{0}$. This last inequality for $A(t)$ can be integrated to yield

$$
-\frac{1}{\epsilon} \int_{t_{0}}^{t} A(s) d s \leq-\kappa \cdot\left(t-t_{0}\right) / \epsilon \text { for } t \geq t_{0}
$$

and then the monotonicity of the exponential function along with (4.12), (4.9), and (4.10) yield

$$
\left|\frac{Y(t)-\lambda X(t)}{y_{0}-\lambda x_{0}}\right|=\frac{Y(t)-\lambda X(t)}{y_{0}-\lambda x_{0}} \leq e^{-\kappa \cdot\left(t-t_{0}\right) / \epsilon} \quad \text { for } \quad t \geq t_{0}
$$


in the present case $y_{0}>\lambda x_{0}$. A similar analysis yields this same result (4.13) in the other case $y_{0}<\lambda x_{0}$ with the appropriate value of $\kappa$ given by (4.3).

If $y_{0}>\lambda x_{0}$, then by Theorem $4 Y>0$ and $X<0$ holds, from which $|X|=-X \leq$ $\lambda^{-1}[Y-\lambda X]=\lambda^{-1}|Y-\lambda X|$ and $|Y|=Y \leq Y-\lambda X=|Y-\lambda X|$. These inequalities, along with (4.13), yield the stated results of (4.2)-(4.4) in the case $y_{0}>\lambda x_{0}$. In the other case $y_{0}<\lambda x_{0}$, by Theorem $4 Y<0$ and $X>0$ holds, from which $|X|=$ $X \leq \lambda^{-1}[-Y+\lambda X]=\lambda^{-1}|Y-\lambda X|$ and $|Y|=-Y \leq \lambda X-Y=|Y-\lambda X|$. These inequalities with (4.13) yield (4.2)-(4.4) if $y_{0}<\lambda x_{0}$, and this completes the proof of the estimates (4.2) with the constants $\xi$ and $\eta$ given as in (4.4).

Note that the analysis also yields useful results if the initial state is in the second quadrant of the phase plane.

Estimates of the form (4.2) follow from general considerations related to the Center Manifold Theorem (cf. Perko [4]), but the present proof is convenient to provide explicit constants such as those of (4.3) and (4.4).

Acknowledgments. We thank a referee for helpful comments on an earlier version of this paper.

\section{References}

1. H. E. Kimmel, Variable speed turbine generators in LNG liquefaction plants. In: GASTECH 96: 17th International LNG/LPG/Natural Gas Conference \& Exhibition, Vol.2, 3-6 December, Vienna, Austria, 1996.

2. _ Speed controlled turbine expanders in hydrocarbon liquefaction processes, Internat. J. of Hydrocarbon Engrg. 1 (1997), 36-40.

3. ㄴ Hydraulic performance of speed controlled turbines for power recovery in cryogenic and chemical processing, World Pumps, Elsevier, Oxford, England, June 1997, pp.46-49.

4. L. Perko, Differential Equations and Dynamical Systems, Springer-Verlag, New York, 1996.

5. D. R. Smith, Singular Perturbation Theory, Cambridge University Press, Cambridge, England, 1985.

6. A. J. Stepanoff, Centrifugal and Axial Flow Pumps, 2d ed., Wiley, 1957.

Department of Mathematics, University of California at San Diego, La Jolla, CA 92093

Research and Development, Cryodynamics Division, EBARA International Corp., 350 SaLOMON CIRCLE, SPARKS, NV 89434 